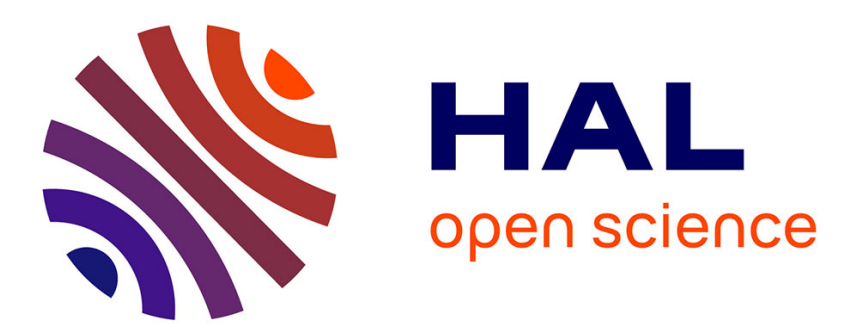

\title{
The Wavelet Response as a Multiscale Characterization of Scattering Processes at Granular Interfaces
}

Yves Le Gonidec, Dominique Gibert

\section{To cite this version:}

Yves Le Gonidec, Dominique Gibert. The Wavelet Response as a Multiscale Characterization of Scattering Processes at Granular Interfaces. 2005. hal-00008567

\section{HAL Id: hal-00008567 https://hal.science/hal-00008567}

Preprint submitted on 13 Sep 2005

HAL is a multi-disciplinary open access archive for the deposit and dissemination of scientific research documents, whether they are published or not. The documents may come from teaching and research institutions in France or abroad, or from public or private research centers.
L'archive ouverte pluridisciplinaire HAL, est destinée au dépôt et à la diffusion de documents scientifiques de niveau recherche, publiés ou non, émanant des établissements d'enseignement et de recherche français ou étrangers, des laboratoires publics ou privés. 


\title{
The Wavelet Response as a Multiscale Characterization of Scattering Processes at Granular Interfaces
}

\author{
Yves Le Gonidec ${ }^{\mathrm{a}}$ and Dominique Gibert ${ }^{\mathrm{b}, *}$, \\ ${ }^{a}$ Géosciences Azur (CNRS/INSU UMR 6526), Observatoire Océanologique de \\ Villefranche-sur-Mer, France. \\ ${ }^{\mathrm{b}}$ Géosciences Rennes (CNRS/INSU UMR 6118), Université Rennes 1, Bât. 15 \\ Campus de Beaulieu, 35042 Rennes cedex, France.
}

\begin{abstract}
We perform a multiscale analysis of the backscattering properties of a complex interface between water and a layer of randomly arranged glass beads with diameter $D=1 \mathrm{~mm}$. An acoustical experiment is done to record the wavelet response of the interface in a large frequency range from $\lambda / D=0.3$ to $\lambda / D=15$. The wavelet response is a physical analog of the mathematical wavelet transform which possesses nice properties to detect and characterize abrupt changes in signals. The experimental wavelet response allows to identify five frequency domains corresponding to different backscattering properties of the complex interface. This puts quantitative limits to the validity domains of the models used to represent the interface and which are flat elastic, flat visco-elastic, rough random half-space with multiple scattering, and rough elastic from long to short wavelengths respectively. A physical explanation based on Mie scattering theory is proposed to explain the origin of the five frequency domains identified in the wavelet response.
\end{abstract}

Key words: Multiscale, Multiple scattering, Granular medium, Wavelet transform, Coda, Mie scattering

PACS: 43.60.+d, *43.40.L, 43.58.+z, 45.70.-n, 46.40.Cd, 46.65.+g

* Corresponding author.Tel.:(+33/0) 2232360 91; Fax: (+33/0) 223236090

Email addresses: legonidec@geoazur.obs-vlfr.fr (Yves Le Gonidec), dominique.gibert@univ-rennes1.fr (Dominique Gibert).

Preprint submitted to Ultrasonics 9 September 2005 


\section{Introduction}

The concept of interface plays a key role in many imaging methods using backscattered waves, and most reflexion techniques (e.g. seismics) are based on models where quasi-homogeneous domains are separated by sharp interfaces where the physical properties of the medium change abruptly [e.g. Miller et al., 1987]. These models are used to reproduce the data as a superimposition of reflected echoes coming from interfaces whose number, position and strength are to be determined. The determination of the position of the interfaces needs an accurate large-scale velocity model whose inversion is recognized to be a highly non-linear tomography problem [Tarantola, 1987; Jin et al., 1992]. This stage of imaging is performed with a smooth model of the distribution of the physical properties, also called the macro-model [Bunks et al., 1995; Billette and Lambaré, 1998; Lambaré et al., 2004], which represents the large-scale heterogeneities of the medium. The macro model is completed by an interface skeleton representing the support of the abrupt impedance contrasts located at the limits of the domains and producing the reflected echoes forming the data. In most approaches, these abrupt impedance contrasts are represented by step-like functions (i.e. the Heaviside distribution). The parameters (i.e. compressional and shear velocities) of the macro-model are adjusted in order to fit with the arrival times and the interface skeleton is used to fit with the sign and the amplitude of the echoes forming the data [Thierry et al., 1999a; 1999b]. A more sophisticated macro model is eventually used to account for the attenuating visco-elastic properties of the media and the dispersion of the body waves [e.g. Ribodetti et al., 2000].

Many situations exist where the model described above is insufficient to correctly account for the reality. In particular, the concept of sharp and step-like interfaces may be strongly inadequate to represent the transition between bodies of heterogeneous material. Geophysical examples are volcanoes, the weathered subsurface [Exon et al., 1998; Vanneste al., 2001], and the shallow layers of sediments forming the seafloor [De Moustier et al., 1993; Augustin et al., 1996]. Ultrasonic imaging in both medical applications and non-destructive testing $(\mathrm{NdT})$ are also faced with pitfalls of the step-like interface concept at transitions involving small-scale heterogeneous media like bones, fat and composite material. A first way to tackle with these difficulties is to use rough-interface models where the transition surface between homogeneous bodies with differing properties is given a rough topography [e.g. Voronovich, 1994] with possibly a fractal geometry [Shepard et al., 1999; Gautier and Gibert, 2004]. However, the rough-interface concept does not generally consider the heterogeneous nature of the material on both sides of the transition, and further sophistications of the interface model would be necessary to properly account for the complex wave phenomena occurring in the vicinity of the interface. A number of questions then arise: In which wavelength range is the step-like 
concept still valid to represent the transition between domains of highly heterogeneous material ? At which wavelengths does the step-like model cease to be valid ? Which kind of more sophisticated models should be used instead?

In the present study, we present and discuss experimental results designed to provide both qualitative and quantitative data about the conceptual modeling of an interface corresponding to the transition between water and a dense layer of randomly arranged glass beads. Both the experiments and the analysis are based on the wavelet response method introduced in a previous paper [Le Gonidec et al., 2002]. In this previous study, we show that the continuous wavelet transform obtained by convolving a signal with a family of constantshape wavelets (see Holschneider [1995] for an introduction) may be physically extended to the wavelet response where a family of wavelets are propagated (i.e. NOT convolved) through the medium to be analyzed. We also show that the wavelet response is equivalent to the wavelet transform of the reflectivity function under the first Born approximation. Consequently, the interesting properties of the wavelet transform concerning the characterization of abrupt changes in signals [e.g. Mallat and Hwang, 1992; Herrmann, 1994; Alexandrescu et al., 1995, 1996] are retrieved in the wavelet response which may be used to remotely analyse the multiscale structure of acoustical interfaces [Walden and Hosken, 1986; Herrmann, 1996; Wapenaar, 1998, 1999; Marsan and Bean, 1999].

This paper continues with a brief presentation of the continuous wavelet transform and its extension to the wavelet response method. Then, the experimental setup is detailed and the experimental wavelet response of the surface of a thick layer of glass bead is presented. In a next section, the wavelet response is analyzed in the framework of the singularity characterization toolbox developed for the continuous wavelet transform [Mallat and Hwang, 1992]. In a last section, we discuss the wave phenomena relevant in the different wavelength bands identified in the wavelet response of the interface. Our physical interpretation is based on the microscopic Mie scattering occurring in the heterogeneous medium.

\section{Analyzing method: the wavelet response}

The wavelet response has been introduced in details by Le Gonidec et al. [2003], and we only recall the main steps of its derivation from the classical continuous wavelet transform which consists in convolving a signal with a family of constant-shape analyzing wavelets [Alexandrescu et al., 1995; Holschneider, 1995]. The wavelet family is obtained by dilating an analyzing wavelet 
$g(t)$

$$
\mathcal{D}_{a} g(t) \equiv \frac{1}{a} g\left(\frac{t}{a}\right),
$$

where $\mathcal{D}_{a}$ represents the dilation operator indexed by the dilation $a>0$ which is inversely proportional to the frequency. The wavelets obtained for a dilation range $a_{\min } \leq a \leq a_{\max }$ have the same shape and constitute a wavelet family spanning a wide wavelength range well-adapted to study multiscale wave phenomena [Le Gonidec et al., 2002, 2003]. The analyzing wavelet must be a time-localized oscillating function with a band-pass spectrum and, at least, a zero-order vanishing moment. For instance, the well-known Ricker source function [Jannaud et al., 1991; Hoshiba, 2000] frequently used in seismic modeling is an acceptable analyzing wavelet $g$.

The wavelet transform is obtained by convolving the whole wavelet family with the analyzed signal, $s(t)$,

$$
\mathcal{W}[g, s](b, a) \equiv\left(\mathcal{D}_{a} g * s\right)(b),
$$

where $*$ is the convolution operator and $b$ is a translation parameter. The main property needed in the present study is the covariance of the wavelet transform which indicates that the wavelet transform of a dilated function is the wavelet transform of the non-dilated function rescaled on both the $a$ and $b$ axes:

$$
\mathcal{W}\left[g, \mathcal{D}_{\beta} s\right](b, a)=\frac{1}{\beta} \mathcal{W}[g, s]\left(\frac{b}{\beta}, \frac{a}{\beta}\right) .
$$

When applied to an homogeneous function of degree $\alpha \in \mathrm{R}$ such that,

$$
s(\beta x)=\beta^{\alpha} s(x),
$$

equation (3) simplifies into,

$$
\mathcal{W}[g, s](\beta b, \beta a)=\beta^{\alpha} \mathcal{W}[g, s](b, a),
$$

which indicates that the whole wavelet transform of a homogeneous function can be obtained from the wavelet transform taken at a given dilation [Mallat and Hwang, 1992]:

$$
\mathcal{W}[g, s]\left(b, a^{\prime}\right)=\left(\frac{a^{\prime}}{a}\right)^{1+\alpha} \mathcal{D}_{a^{\prime} / a} \mathcal{W}[g, s](b, a),
$$


where the dilation operator is understood to act on the translation variable $b$ only. The geometrical sense of this equation is that the wavelet transform of a homogeneous singularity has the appearance of a cone whose apex points onto the singularity for $a \downarrow 0^{+}$. Equation (6) indicates that the magnitude of the wavelet transform is $\propto a^{\alpha}$ when sampled along the cone lines also called the ridge functions (see Alexandrescu et al. [1995, 1996] and Le Gonidec et al. [2003] for details). The Heaviside distribution $H$ used to represent step-like interfaces as discussed above is homogeneous of degree $\alpha=0$ [Bracewell, 1986], and its wavelet transform has a constant amplitude along the ridge functions.

The wavelet response $\mathcal{R}[g, s]$ is obtained by propagating the wavelet family through the velocity profile $s$ along the raypath,

$$
\mathcal{R}[g, s](b, a) \equiv\left(\mathcal{D}_{a} g \bigotimes s\right)(b)
$$

where $\otimes$ represents the physical propagation inside the medium and $b$ is the two-way flight time. Practically, each line $a=$ constant of $\mathcal{R}[g, s]$ is the rescaled (i.e. multiplied by dilation $a$ ) time-signal of the backscattered waves recorded after the launch of the corresponding member of the wavelet family. Neglecting multiple scattering and assuming a constant density profile, Le Gonidec et al. [2003] show that the wavelet response may be rewritten in a form similar to the classical continuous wavelet transform (2):

$$
\mathcal{R}[g, s](b, a)=\left(\mathcal{D}_{a} g * \frac{\mathrm{d}}{\mathrm{d} z} s[z(t)]\right)(b) .
$$

A comparison of equations (2) and (8) indicates that the wavelet response of the velocity profile $s$ is the wavelet transform of the first spatial derivative of $s$. Consequently, both $\mathcal{W}$ and $\mathcal{R}$ give the same result when applied to $s$ when the wavelets used in $\mathcal{W}$ are the derivatives of those used in $\mathcal{R}$. Several synthetic and experimental examples may be found in Le Gonidec et al. [2003] where similarities and differences between $\mathcal{W}$ and $\mathcal{R}$ are discussed in more details.

An ideal step-like interface is mathematically represented by $s_{H}(z)=s_{0}+$ $\Delta s H(z)$, where $\Delta s$ is the velocity jump at the interface. The synthetic wavelet response of $s_{H}$ is shown in Figure 1(a) where the cone-like structure converging toward the interface is conspicuous. The step-like nature (i.e. $\alpha=0$ ) of the transition may be verified by checking that the magnitude of the wavelet response is constant along the cone lines as shown in Figure 1(b). The characterization of the reflector structure may then simply be inferred from the homogeneity degree $\alpha$ obtained from the ridge functions. This has been assessed from an experimental point of view in an ideal case of planar interfaces [Le Gonidec et al., 2003] and is now assessed from a more complex situation of an interface represented by the upper part of a granular medium. 


\section{Wavelet response of a layer of glass beads}

\subsection{Experimental setup and reference wavelet response}

The experimental setup is immersed in a large water tank and consists in a rigid frame with a top plate holding the source and receiver transducers mounted in the near normal incidence configuration (Figure 2). The transducers beams focus at a common point onto the surface of the target placed on a bottom plate which may be horizontally translated.

The first stage of the experimental procedure is to calibrate the source-receiver chain by constructing a reference wavelet response to be compared with the wavelet response of the layer of glass beads. In the present study, the wavelet response of a step-like interface at normal incidence is used as the reference [Le Gonidec et al., 2003]. This response is constructed by searching the source signals to be used such that the wavelet response recorded for a step-like interface (represented by a flat block of glass) actually corresponds to the theoretical response shown in Figure 1. This is done with a non-linear inverse technique based on simulated annealing and neural network (see Conil et al. [2004] for technical details), and four pairs of transducers with central frequen-

cies $f_{c}=250,500,750$, and $1000 \mathrm{kHz}$ are used to construct a wavelet family with 30 members for central frequencies $200 \mathrm{kHz} \leq f_{c} \leq 1 \mathrm{MHz}$ (Figure 3).

\subsection{Wavelet response of granular media}

In this paper, the target interface is the transition between water and the surface of a layer of glass beads randomly filling a rolling box beneath the transducers (Figure 2). The thickness of the granular medium is taken large enough $(20 \mathrm{~cm})$ to avoid any reflection from the bottom of the box: this is verified by sending the lowest-frequency wavelet and checking that no reflection from the box bottom occurs. The granular is made of monodisperse glass beads immersed in a fluid matrix, i.e. the water of the talk. We determine the bead concentration $\phi \simeq 0.63$ by the volume of water needed to fill the dry medium pores: the result agrees with the concentration found for close random packing of glass beads [Page et al., 1995, 1996]. Highest concentration of about 0.74 should be reached for arranged packings of spherical inclusions [Neser et al., 1997]. The glass beads are mixed into the water in order to suppress air bubbles; the surface of the layer is slightly smoothed to produce a flat reflector at the macroscopic scales and to avoid any ordered arrangement of the spheres [Sheng, 1995].

For each individual wavelet, we stack 1000 individual reflected signals while 
moving the box to define the average wavelet response of the interface. This approach is performed on six quasi-monodisperse glass bead diameters, respectively: $D=0.6 \mathrm{~mm}, 1 \mathrm{~mm}, 2 \mathrm{~mm}, 3 \mathrm{~mm}, 4 \mathrm{~mm}$, and $5 \mathrm{~mm}$. We merge and rescale the six average wavelet responses so obtained to compute the wavelet response for a unique glass bead diameter $D=1 \mathrm{~mm}$ : this equivalent wavelet response is then composed by 57 wavelets spanning the frequency range $[100 \mathrm{kHz} ; 5 \mathrm{MHz}]$ (Figure 4).

\section{Analysis of the wavelet response}

\subsection{Cone-like structure}

A first glance at Figure 4 reveals that the wavelet response of the granular interface dramatically changes throughout the spanned frequency range. The most conspicuous feature is a two-fold behavior of the wavelet response with a clear cone-like structure in the low-frequency domain which suddenly disappears to be replaced by a coda pattern in the high-frequency range. This first analysis shows that the surface of the granular layer is identified as a localized interface at wavelengths $\lambda \geq \sqrt{\pi} D$. The structure of the conical pattern is similar to the one obtained for a step-like interface (Figure 3) and, in particular, their numbers of extrema are the same. This last point confirms the step-like nature of the interface since a change of regularity $\alpha$ would have modified the extrema arrangement by changing their relative amplitude or even changing

their number (see Le Gonidec et al., [2003] for a detailed discussion of these aspects).

The high-frequency range of the wavelet response has a totally different appearance and the absence of a conical pattern indicates that the surface of the layer of glass beads can no more be represented as a localized interface as for the low frequencies. In this domain, most of the reflected waves are part of the coda component which duration indicates backscattered waves coming from deep within the granular medium.

\subsection{Coherent frequency bands}

The analysis can be made more quantitative by examining the variation of the magnitude of the wavelet response along the ridge functions. For this purpose, we have plotted the first 5 ridge functions belonging to the cone in the low-frequency domain. The corresponding ridge paths are numbered and represented by black dots in Figure 4, and the ridge functions are shown 
in Figure 5(b) where the amplitude of the wavelet extrema are plotted as a function of $r=\lambda / D$ in a $\log -\log$ diagram. The average ridge function is also shown in the same Figure as a solid curve.

As can be observed in Figure 5(b), the ridge functions are clearly segmented and five domains labeled from $I$ to $V$ can be recognized. The limits between these domains roughly fall at $\lambda / D=2 \pi, \pi, \pi / 2$ and $2 / \pi$. Excepted for domain III where the ridge functions display a pronounced minimum, the remaining four domains correspond to frequency bands where the ridge functions are linear segments. Domain $I$ corresponds to a linear and horizontal part of the ridge functions. In domain $I I$, the ridge functions are linear with a slope equals to +1 . Domain $I V$ falls in the short-wavelength part of the wavelet response where, again, the ridge functions are linear with a slope equals to +2 in the $\log -\log$ plot. Domain $V$ corresponds to the short-wavelength end of the ridge functions where the energy level is very low with a white-noise appearance. As already noted, domain $I I I$ covers a dilation range where the ridge functions are not linear and where they appear more scattered with a low energy level. It is interesting to note that this intermediate domain corresponds to the disappearance of the main cone around $\lambda / D \simeq \sqrt{\pi}$. The characteristics of these five domains are now discussed in more details.

\subsection{Long (relative) wavelength domains I and II}

Both domains $I$ and $I I$ cover the dilation range $\lambda / D>\pi$ where the wavelet response has a single conical appearance with 5 ridge functions as observed in the reference wavelet response shown in Figure 3. Hence, from this point of view, domains $I$ and $I I$ cannot be distinguished, and only the break of slope observed in the ridge function puts a limit at $\lambda / D=2 \pi$ between these two domains. In both domains, the temporal durations of the reflected wavelets are identical to those of the incident wavelets. This indicates that the granular medium does not modify the shape of the incident wavelets when $\lambda / D>\pi$.

In the $\log -\log$ plot of Figure $5(\mathrm{~b})$, the ridge functions in domain $I(\lambda / D>$ $2 \pi)$ are linear with a slope $\alpha=0$ as theoretically predicted for the wavelet response of a flat elastic interface (Figure 1(b)). Hence, when the incident wavelength is larger than twice the circumference of the glass beads, i.e. when $\lambda / D>2 \pi$, the layer of monodisperse glass beads is equivalent to an elastic homogeneous medium. The amplitude of the ridge function directly gives a constant reflectivity $R=0.34$ at normal incidence.

In domain $I I$ where $2 \pi>\lambda / D>\pi$, the ridge functions remains linear but with a slope $\alpha=+1$. In this domain, the surface of the granular medium may again be considered as step-like since the conical appearance of the wavelet response 
is not modified. However, the $\lambda$ dependance of the reflectivity $R$ indicates that the heterogeneous medium is not equivalent to an elastic homogeneous medium but, instead, is equivalent to a visco-elastic homogeneous medium with a normal reflectivity which linearly decreases from $R=0.34$ to $R=0.15$ as the wavelength shortens.

\subsection{Medium (relative) wavelength domain III}

Domain $I I I$ covers the range $\pi>\lambda / D>\pi / 2$ where the global cone-like pattern of the wavelet response progressively disappears and splits into two sub-conical structures at $\lambda / D \simeq \sqrt{\pi}$ (Figure $5(\mathrm{a})$ ). The reflectivity in domain III appears ill-defined because of some scattering in the data, however the average ridge function possesses a deep minimum $R=0.025$ at $\lambda / D \simeq \sqrt{\pi}$. The coherence of the wavelet response is destroyed when the wavelength of the incident wave becomes smaller than the circumference of the beads, i.e. when $\lambda / D<\pi$. The splitting of the wavelet response creates new ridge functions whose number increases as the wavelength shortens. Meanwhile, as can be observed in Figure 5(b), the amplitude of the ridge functions does not obey a power law as observed in domains $I$ and $I I$. Domain $I I I$ then corresponds to a wavelength range where significant late arrivals appear and make the duration of the back-scattered wavetrains longer than the duration of the incident wavelets. Domain $I I I$ is a transition region where the surface of the granular medium may no more be considered as a step-like interface.

\subsection{Short (relative) wavelength domains $I V$ and $V$}

Domains $I V$ and $V$ correspond to the shorter wavelengths used in the experiment and such that $\lambda / D<\pi / 2$. In these domains, the conical structure of the wavelet response is totally blurred and most energy is carried by a strong and well-developed coda (Figure 5(a)). Numerous ridge functions are found in the wavelet response with an amplitude higher than the one of the first five ridge functions $(1-5)$ observed in all domains. This phenomenon is due to intense multiple scattering of the incident wave with the small-scale structure of the granular medium. The plot of the ridge functions displayed in Figure 5(b) shows that the ridge functions are linear with different slopes in two frequency ranges defining domains $I V$ and $V$. Domain $I V$ corresponds to $\pi / 2>\lambda / D>2 / \pi$ where the ridge functions has a slope $\alpha=+2$ in the $\log -\log$ plot, i.e. with a strong $\lambda$ dependance of the reflectivity $R$. Domain $V$ corresponds to $\lambda / D<2 / \pi$ where the ridge functions appear scattered with an average slope $\alpha=0$. 


\section{Discussion of Wave Phenomena}

\subsection{Propagation-to-diffusion transition}

The five wavelength domains recognized in the experimental wavelet response (Figure 5) have very different reflectivity characteristics corresponding to different wave phenomena at work. A conspicuous feature is the rather sharp transition which exists between the reflectivity domains. Indeed, the $r$ dependance of the reflectivity derived from the ridge functions (Figure 5(b)) reveals that, as $r$ diminishes, the reflectivity departs more and more from the $r$-independant one corresponding to a flat elastic interface as observed in domain $I$. This indicates that, the shorter the wavelength the greater the perturbations of the re-radiated wavefield by the granular medium.

A first quantitative assessment of the perturbations produced by the granular medium can be derived from the ratio between the energy, $E_{b}$, of the ballistic part of the wavelet response and the energy, $E_{c}$, of the late arrivals forming the coda part (Figure 5(c)). The duration of the ballistic part is taken equal to the theoretical duration of the incident wavelets, i.e. the duration of the conical pattern of the reference wavelet response shown in Figure 3. From this definition, we observe that the wavelet response in domains $I$ and $I I$ is almost totally restricted to the ballistic response, contrarily to what occurs in domains $I I I, I V$ and $V$ where the coda part is more and more present (Figures 4 and 5(a)).

The ballistic-to-coda energy ratio curve in Figure 5(c) clearly indicates that domain $I I I$ is an important transition where the relative energy of the coda dramatically increases by more than one order of magnitude as $r$ gets smaller. In domains $I$ and $I I$, the ballistic energy $E_{b}>10 E_{c}$, and the scattering phenomena are weak. Conversely, the coda energy $E_{c}>10 E_{b}$ in domains $I V$ and $V$ indicates that most of the wavelet response comes from multiple scattering produced by the glass beads.

\subsection{Small-scale Mie scattering}

The multiple scattering effects observed at the macroscopic scales result from wave phenomena occurring at microscopic length scales corresponding to the diameter of the glass beads. We shall now discuss the five frequency bands identified in the wavelet response in terms of wave scattering produced by a single scatterer.

Assuming an incident plane wave traveling in the $\theta=\pi$ direction, the Mie 
scattering theory [Goodman and Stern, 1962] defines the scattering amplitude $|f(\theta)|^{2}$ of the wave re-radiated by a spherical scatterer, where $\theta$ is the scattering angle. The 2-D directivity patterns of a spherical scatterer have been computed for the five frequency bands of interest using the physical properties of the glass: density $=2560 \mathrm{~kg} \cdot \mathrm{m}^{-3}$, compressional velocity $=5500 \mathrm{~m} . \mathrm{s}^{-1}$ and shear velocity $=3400 \mathrm{~m} \cdot \mathrm{s}^{-1}$. The results are plotted in Figure 6(a). The origin of the axis (black dot) corresponds to the center of the spherical scatterer. In particular, $\theta=0$ and $\theta=\pi$ correspond to the longitudinal back(i.e. leftward) and forward-scattering (i.e. rightward) directions respectively, transverse scattering corresponds to $\theta=\pi / 2$.

The ratio between the back- and forward-scattering amplitudes is shown in Figure 6(b). This ratio quantifies the amount of energy reflected by the glass bead compared to the energy scattered in the incident direction. From this curve, we observe that the low-frequency bands $I$ and $I I$ correspond to a dominant back-scattering regime where the back-scattered energy is about one order of magnitude larger than the energy re-radiated in the forward direction.

The total scattering cross section, normalized by the geometrical cross section of a glass bead, characterizes the extinction coefficient of an isolated scatterer,

$$
\sigma_{t}=\int|f(\theta)|^{2} d(\theta)
$$

In the frequency band $I$, the extinction coefficient follows a Rayleigh scattering law $\propto \lambda^{-4}$ [Ishimaru, 1978] (Figure 7). This indicates a single scattering regime inside the granular medium when $\lambda / D>2 \pi$.

The ratio between the transverse- and the longitudinal-scattering amplitudes is shown in Figure 6(c): it indicates the amount of energy laterally scattered by the glass bead compared to the energy scattered in the dominant (i.e. backward or forward) longitudinal direction. Both the back-to-forward and the transverse-to-longitudinal ratios reveal that the medium-frequency band III corresponds to a dramatic decrease of the backscattering energy and to a sharp increase of the transverse energy. The $r=2$ value corresponds to an equilibrium between back-scattering and forward-scattering. This value also corresponds to the frequency where the energy in the transverse direction is maximum (Figure 6(c)). The strong decrease of reflectivity observed in band III of the wavelet response may thus be explained by the fact that a significant amount of the energy of the incident wave is re-radiated perpendicularly to the incident direction.

The high-frequency bands $I V$ and $V$ correspond to a dominant forwardscattering regime where a large part of the scattered wave is directed deeper in the layer of beads. In this situation, the scattered waves follow long tor- 
tuous paths in the heterogeneous medium and multiple scattering dominates. The waves eventually re-emerge from the bead layer in the back-scattering direction and contribute to the formation of the strong coda observed in the wavelet response (Figures 5(a) and 4).

The scattering phenomena occurring at the scale of a single glass bead are in a total agreement with the frequency bands observed in wavelet response of the whole layer of glass beads. This confirms that the macroscopic response of the heterogeneous medium is controlled by cooperative scattering occurring at microscopic scales. It is interesting to note that the longitudinal cross-section ratio is similar to the ridge function of the granular medium (Figure 5(b) compared to Figure 6(b)): the global acoustic response of monodisperse spherical inclusions can be interpreted from an isolated inclusion. The experiment limit $r=\pi / 2$ corresponds to a resonance inside the glass beads, as predicted by the Mie theory.

\section{Conclusion}

The wavelet-response method [Le Gonidec et al., 2002, 2003; Conil et al., 2004] used in the present study allows to obtain a coherent acoustical response of a target in a large frequency range. This range may be further increased by rescaling and merging the individual responses of several layers of glass beads with different diameters. The resulting wavelet response then spans a 5-octaves frequency range, and the dimensionless parameter $r=\lambda / D$ varies from $r=0.3$ to $r=15$ (Figures 4 and 5).

Five $r$-ranges are identified in the wavelet response with limits at (roughly) $r=2 \pi, r=\pi, r=\pi / 2$ and $r=2 / \pi$. The wavelet response presents a coherent structure with flat ridge functions when $r>2 \pi$. It is only in this range $I$ that the granular medium is equivalent to a static effective medium with frequency-independent physical properties. In range $I I, 2 \pi>r>\pi$, the wavelet response still presents a simple coherent structure but with a frequency-dependent amplitude which corresponds to an increasing departure from the Rayleigh scattering almost satisfied in range $I$ (Figure 7).

In the higher frequency domain, three $r$-ranges, $I I I, I V$ and $V$, are identified where multiple scattering phenomena play the dominant role. The main characteristic of band $I I I$ is a pronounced decrease of the energy of the backscattered wavefield, and we show that this corresponds to a dominant lateral Mie scattering at the scale of a single glass bead (Figure 6(b)). Band $I V$ corresponds to the domain where a strong coda wave exists in the wavelet response and to a dominant forward-scattering at the scale of a single scatterer. 
The experiments discussed in the present paper show that an heterogeneous medium made of a random packing of scatterers has a macroscopic acoustical response which strongly depends on the incident wavelength. Whatever complicated, we show that this response may be decomposed into five sub-domains, each with a dominant Mie scattering regime at the scale of a single scatterer. From the wavelet response experiments on granular media, we are able to define the wavelength range where the interface can be assimilated to a step-like discontinuity or to a highly heterogeneous material. At low frequencies, when $\lambda / D>\pi$, granular media can be replaced by equivalent homogeneous media: most of deep structure imaging from seismics are based on this assumption. At high frequencies, when $\lambda / D<\pi / 2$, the granular medium is a complex medium where multiple scattering exists: multi-physics modeling is then necessary to perform seismic imaging of complex interfaces such as subsurface imaging and seafloor characterization which involve high frequency source signals.

\section{Acknowledgements}

We thank Frédéric Conil for his help when constructing the wavelet family and Michel Lemoine who helped us in designing the experimental setup. This work is financially supported by the CPER "Sismique" from IFREMER and by the CNRS and ANDRA through the GdR FORPRO (Research action 99.II) and corresponds to GdR FORPRO contribution number 2004/14 A.

\section{References}

Alexandrescu, M., D. Gibert, G. Hulot, J.-L. Le Mouël, and G. Saracco, Detection of geomagnetic jerks using wavelet analysis, J. Geophys. Res., 100, $12557-12572,1995$.

Alexandrescu, M., D. Gibert, G. Hulot, J.-L. Le Mouël, and G. Saracco, Worldwide wavelet analysis of geomagnetic jerks, J. Geophys. Res., 101, 2197521994, 1996.

Augustin, J.M., R. Le Suave, X. Lurton, M. Voisset, S. Dugelay, and C. Satra, Contribution of the multibeam acoustic imagery to the exploration of the sea-bottom, Marine Geophys. Res., 18, 459-486,1996.

Billette, F., and G. Lambaré, Velocity macro-model estimation from seismic reflection data by stereotomography, Geophys. J. Int., 135, 671-690, doi:10.1046 /j.1365-246X.1998.00632.x, 1998. 
Bracewell, R.N., The Fourier transform and its applications, 486 pp., McGrawHill, New York, 1986.

Bunks, C., Fatimetou M. Saleck, S. Zaleski, and G. Chavent, Multiscale seismic waveform inversion, Geophysics, 60, 1457-1473, 1995.

Conil, F., D. Gibert, and F. Nicollin, Nonlinear synthesis of input signals in ultrasonic experimental setups, J. Acoust. Soc. Am., 115, 246-252, 2004.

De Moustier, C., and H. Matsumoto, Seafloor acoustic remote sensing with multibeam echo-sounders and bathymetric sidescan sonar systems, Marine Geophys. Res., 15, 27-42, 1993.

Exon, N.F., G.R. Dickens, J.-M. Auzende, Y. Lafoy, P.A. Symonds, and S. Van de Beuque, Gas hydrates and free gas on the Lord Howe Rise, Tasman Sea, PESA Journal, 26, 148-158, 1998.

Gautier, S., and D. Gibert, Scattering from a fractal surface: Acoustical experiments and comparison with near-nadir models, Icarus, 167\%, 453463, 2004.

Goodman, R.R., and R. Stern, Reflection and transmission of sound by elastic spherical shells, J. Acoust. Soc. Am., 343, 338-344, 1962.

Herrmann, F.J., Scaling of the pseudo primary analyzed by the wavelet transform, Expanded Abstracts of the 64th meeting of SEG, Tulsa, 1049-1052, 1994.

Herrmann, F.J., and J.J. Staal, Waves in scaling media, the implication of non-differentiability, 58th annual EAGE conference, D.J. Feenstra (ed), Amsterdam, abstract nr. C007, 1996.

Holschneider, M., Wavelets: An Analysis Tool, 423 pp., Clarendon, Oxford, England, 1995.

Hoshiba, M., Large fluctuation of wave amplitude produced by small fluctuation of velocity structure, Physics of the Earth and Planetary Interiors, 120, 201-217, 2000.

Ishimaru, A., Wave propagation and scattering in random media, IEEE Press, 1978.

Jannaud, L.R., P.M. Adler, and C.G. Jacquin, Spectral analysis and inversion of codas, J. Geophys. Res., 96(B11), 18215-18231, 1991.

Jin, S., R. Madariaga, J. Virieux, and G. Lambaré, Two-dimensional asymptotic iterative elastic inversion, Geophys. J. Int., 108, 575-588, 1992.

Lambaré, G., M. Alerini, R. Baina, and P. Podvin, Stereotomography: a semi- 
automatic approach for velocity macromodel estimation, Geophys. Prosp., 52, 671-681, 2004.

Le Gonidec, Y., D. Gibert, and J.-N. Proust, Multiscale analysis of waves reflected by complex interfaces: Basic principles and experiments, J. Geophys. Res., 107(B9), 2184, doi:0.10029/2001JB000558, 2002.

Le Gonidec, Y., F. Conil, and D. Gibert, The wavelet response as a multiscale NDT method, Ultrasonics, 41, 487-497, 2003.

Mallat, S., and W.L. Hwang, Singularity detection and processing with wavelets, IEEE Trans. Inf. Theory, 38, 617-643, 1992.

Marsan, D., and C.J. Bean, Multiscaling nature of sonic velocities and lithology in the upper crystalline crust: Evidence from the KTB Main Borehole, Geophys. Res. Lett., 26, 275-278, 1999.

Miller, D., M. Oristaglio, and G. Beylkin, A new slant on seismic imaging: Migration and integral geometry, Geophysics, 52, 943-964, 1987.

Neser, S., C. Bechinger, P. Leiderer, and T. Palberg, Finite-size effects on the closest packing of hard spheres,Phys. Rev. Lett., 7912, 2348-2351, 1997.

Page, J.H., H.P. Schriemer, A.E. Bailey, and D.A. Weitz, Experimental test of the diffusion approximation for multiply scattered sound, Phys. Rev. E, 523, 3106-3114, 1995.

Page, J.H., P. Sheng, H.P. Schriemer, I. Jones, X. Jing, and D.A. Weitz, Group velocity in strongly scattering media, Science, 271, 634-637, 1996.

Ribodetti, A., S. Operto, J. Virieux, G. Lambaré, H. P. Valéro, and D. Gibert, Asymptotic viscoacoustic diffraction tomography of ultrasonic laboratory data: A tool for rock properties analysis, Geophys. J. Int., 140, 324-340, 2000.

Sheng, P., Introduction to Wave Scattering, Localization and Mesoscopic Phenomena, Academic Press, Boston, 1995.

Shepard, M.K., Campbell, B.A., Radar scattering from a self-affine fractal surface: Near-nadir regime. Icarus, 141, 156171, 1999.

Tarantola, A., Inverse Problem Theory Elsevier, New York, 1987.

Thierry, P., G. Lambaré, P. Podvin, and M. Noble, 3-D preserved amplitude prestack depth migration on a workstation, Geophysics, 64, 222-229, 1999a.

Thierry, P., S. Operto, and G. Lambaré, Fast 2D ray-Born inversion/migration in complex media, Geophysics, 64, 162-181, 1999b. 
Vanneste, M., M. De Batist, A. Golmshtok, A. Kremlev, and W. Versteeg, Multi-frequency seismic study of gas hydrate-bearing sediments in Lake Baikal, Siberia, Marine Geology, 172, 1-21, 2001.

Voronovich, A.G., Wave Scattering from Rough Surfaces, Spinger-Verlag, Berlin, 1994.

Walden, A.T., and J.W.J. Hosken, The nature of the non-Gaussianity of primary reflection coefficients and its significance for deconvolution, Geophys. Prosp., 34, 1038-1066, 1986.

Wapenaar, K., Amplitude-variation with angle behavior of self-similar interface, Geophysics, 64, 1928-1938, 1999.

Wapenaar, K., Seismic reflection and transmission coefficients of a self-similar interface, Geophys. J. Int., 135, 585-594, 1998.

Wysession, M.E., E.A. Okal, and C.R. Bina, The structure of the core-mantle boundary from diffracted waves, J. Geophys. ResGeophysics, 97, 8749-8764, 1992. 


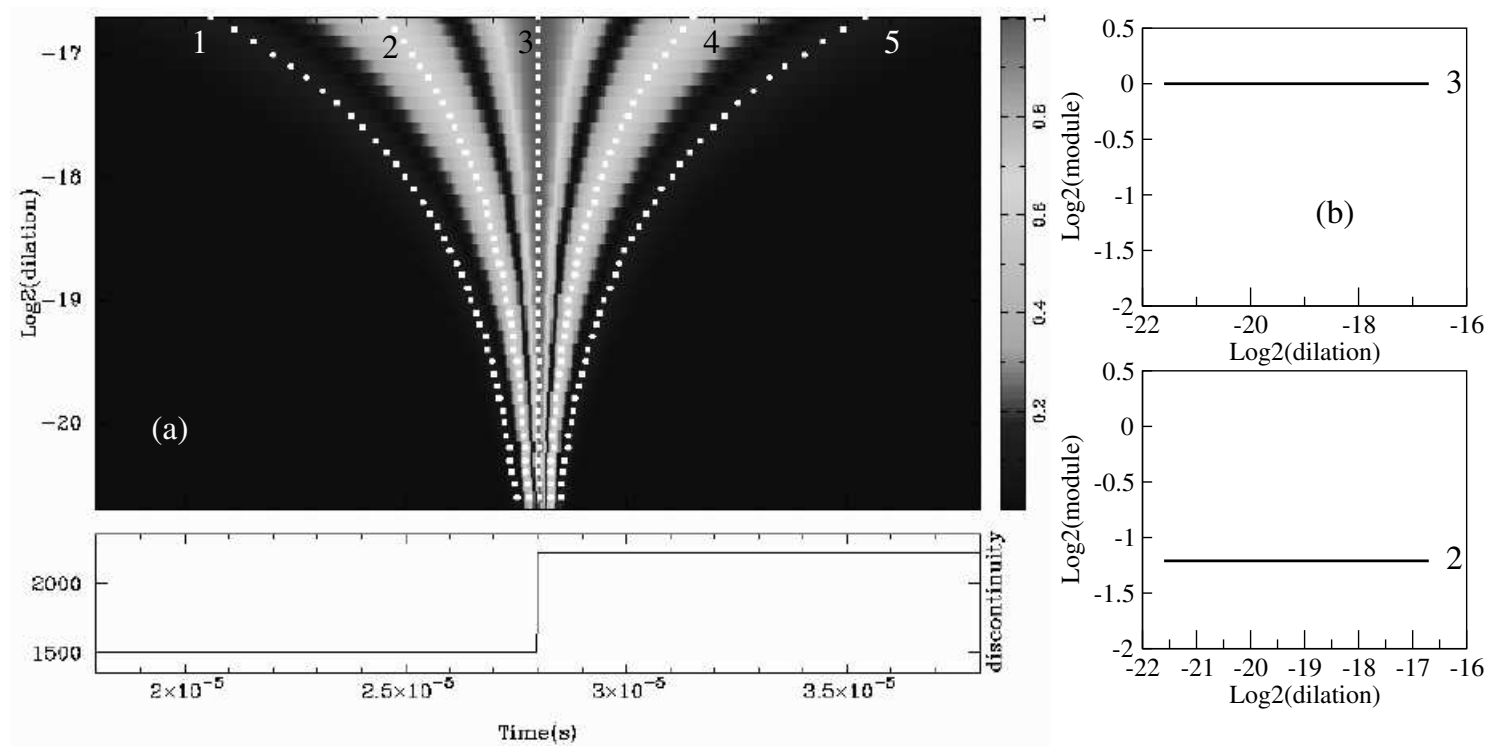

Fig. 1. a: (up) Wavelet response of a homogeneous velocity profile for a Heaviside discontinuity (down). The black dots follow the ridge functions corresponding to high signal-to-noise ratios. b: the ridge functions, numbered 1 and 2 respectively, are straight lines in a $\log -\log$ diagram. The slopes equal the homogeneity degree $\alpha$ of the velocity profile, i.e. 0 in the present case of a Heaviside discontinuity. 


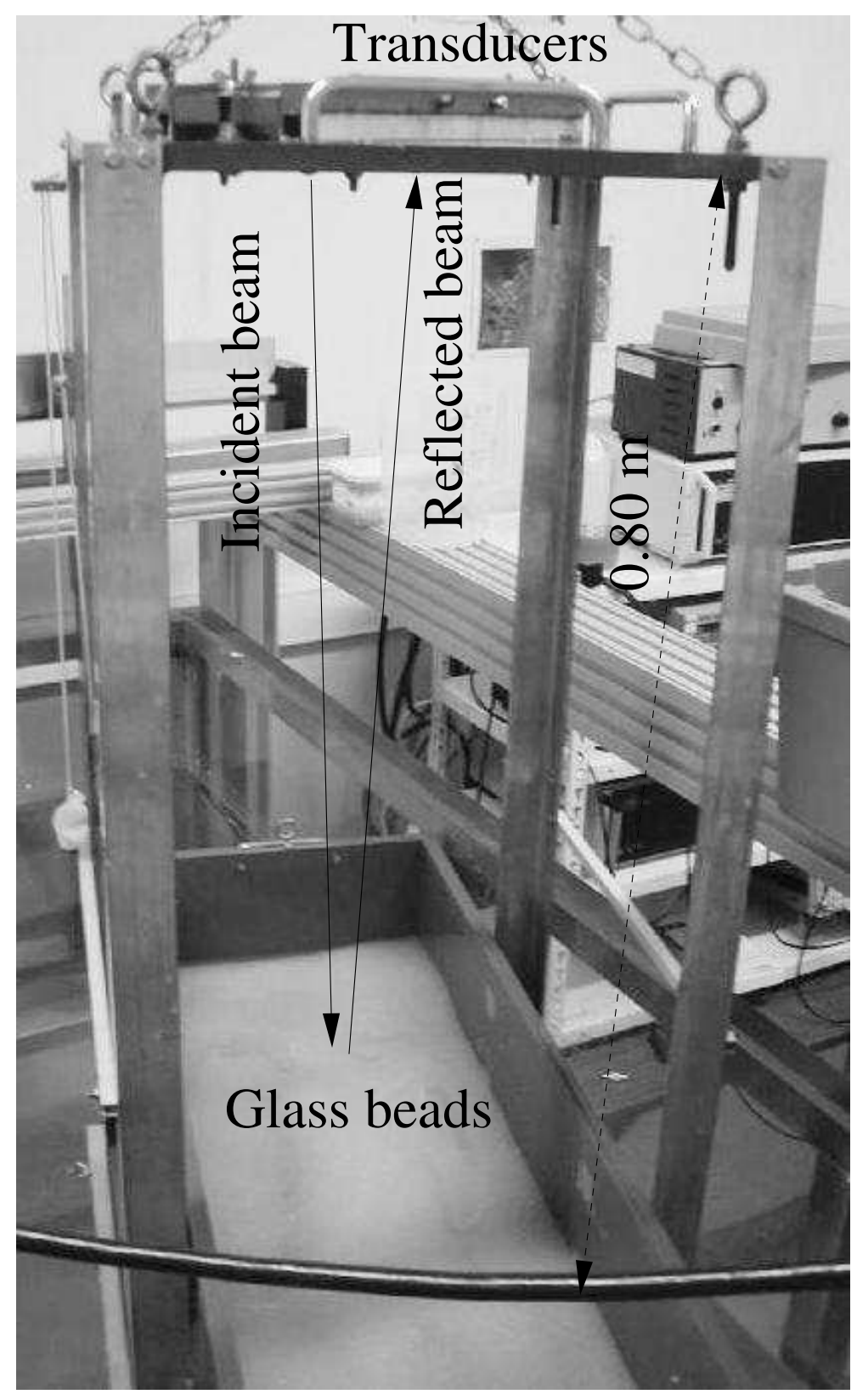

Fig. 2. The experimental setup is composed of a frame whose top holds two piezoelectric transducers. The box containing the glass beads is placed at the bottom of the frame and can be horizontally translated while stacking the reflections from the layer of glass beads in order to record the average reflected field. 


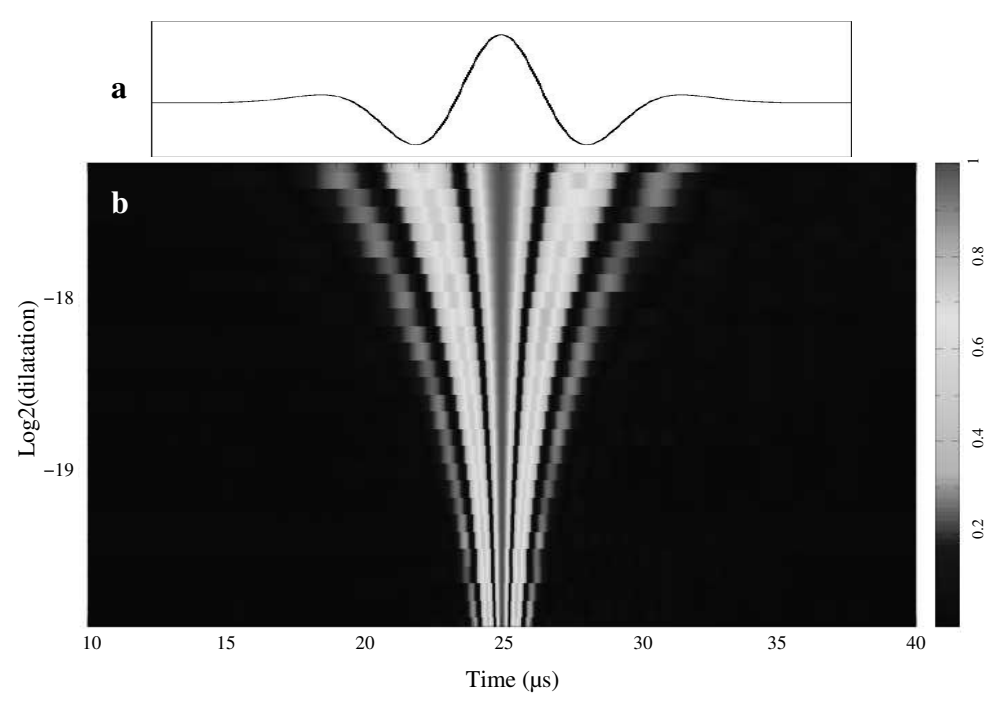

Fig. 3. a : The analytical wavelet used for the experiments is the fourth derivative of a Gaussian : $d^{4}\left(e^{\left.-t^{2}\right) / d t^{4}}\right.$. b : Modulus of the experimental reference wavelet response obtained by propagating the source signals through water. The time-dilation $a=f_{c}^{-1}$ where $f_{c}$ is the main frequency of the wavelets which are dilated versions of the fourth derivative of a Gaussian. This reference wavelet response corresponds to the reflection onto a flat interface with a Heaviside-like discontinuity. 


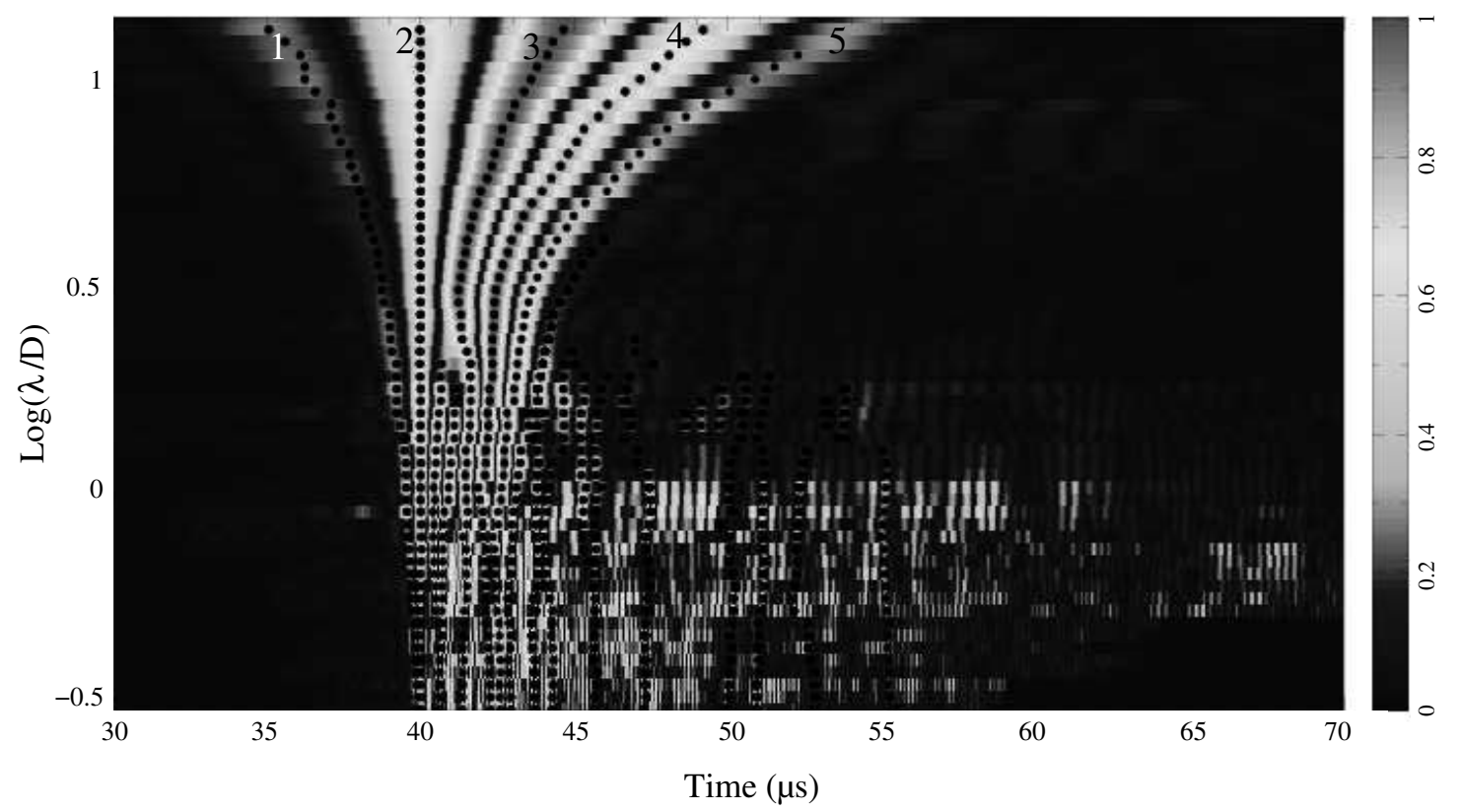

Fig. 4. Modulus of the composite experimental wavelet response rescaled for glass beads with $D=1 \mathrm{~mm}$ (see text for details). The black dots represent the lines of maxima associated with the ridge functions numbered in Figure 5(b). 


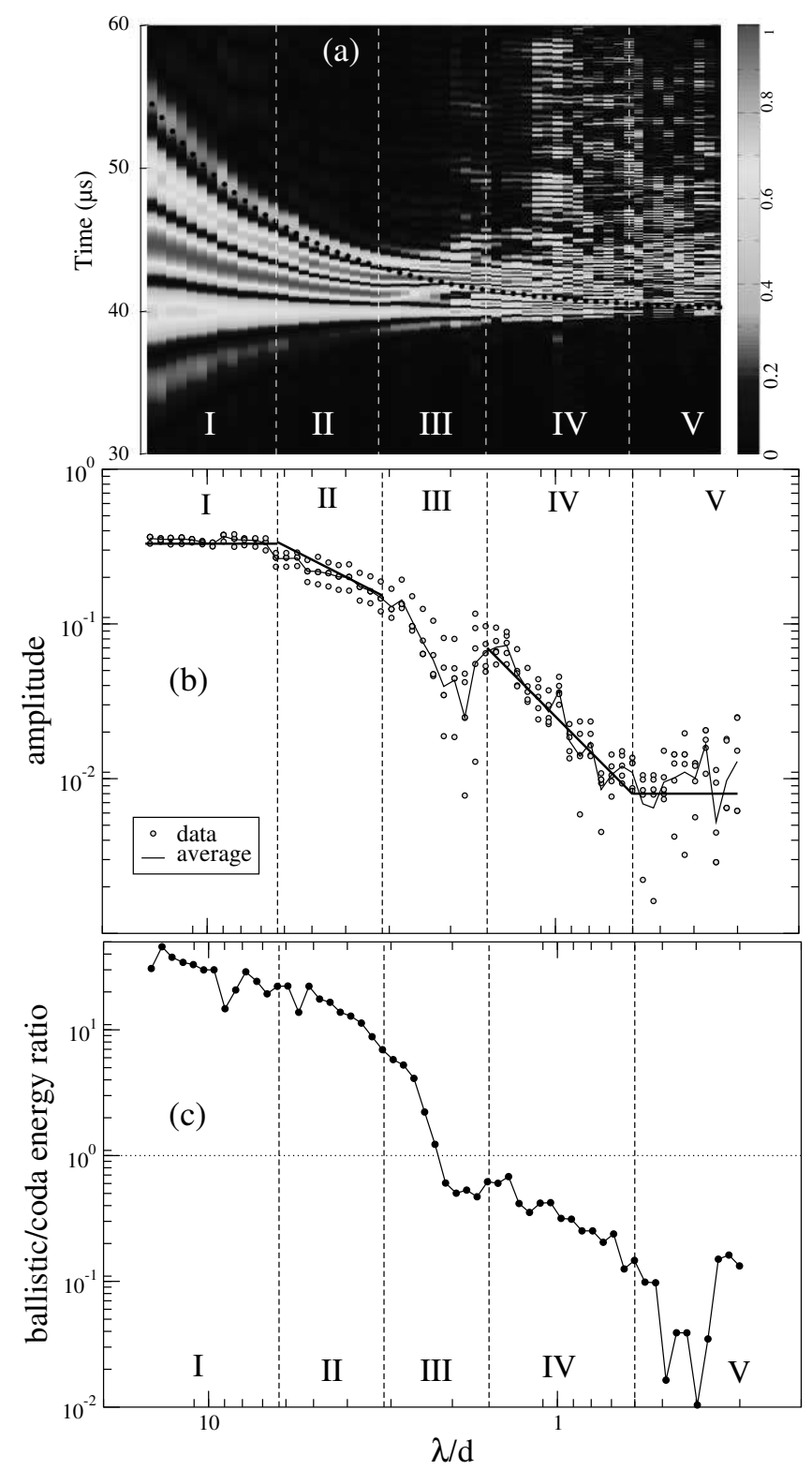

Fig. 5. a: Modulus of the composite experimental wavelet response (see text and Figure 4 for details) represented in a log-linear diagram. The black dots represent the temporal duration of each wavelet: below and above this line are the ballistic and coda components of the reflected wavelets, respectively. b: Experimental ridge functions (symbols) plotted in a $\log$ - log diagram and corresponding to the four labeled lines of maxima extracted from the experimental wavelet response. Also shown is the average ridge function (solid line), mostly linear in 4 frequency bands represented by straight segments with slopes equal to $0,+1,+2$, and 0 from right to left respectively. c: Ratio between the energy of the ballistic component and the energy of the coda extracted from the experimental wavelet response (see Figure $5(\mathrm{a})$ ). In the bands $I$ and $I I$, the propagation regime dominates. The band $I I I$ corresponds to the transition between the propagation to the diffusion regime (bands $I V$ and $V$ ) of the waves reflected by the glass beads. 

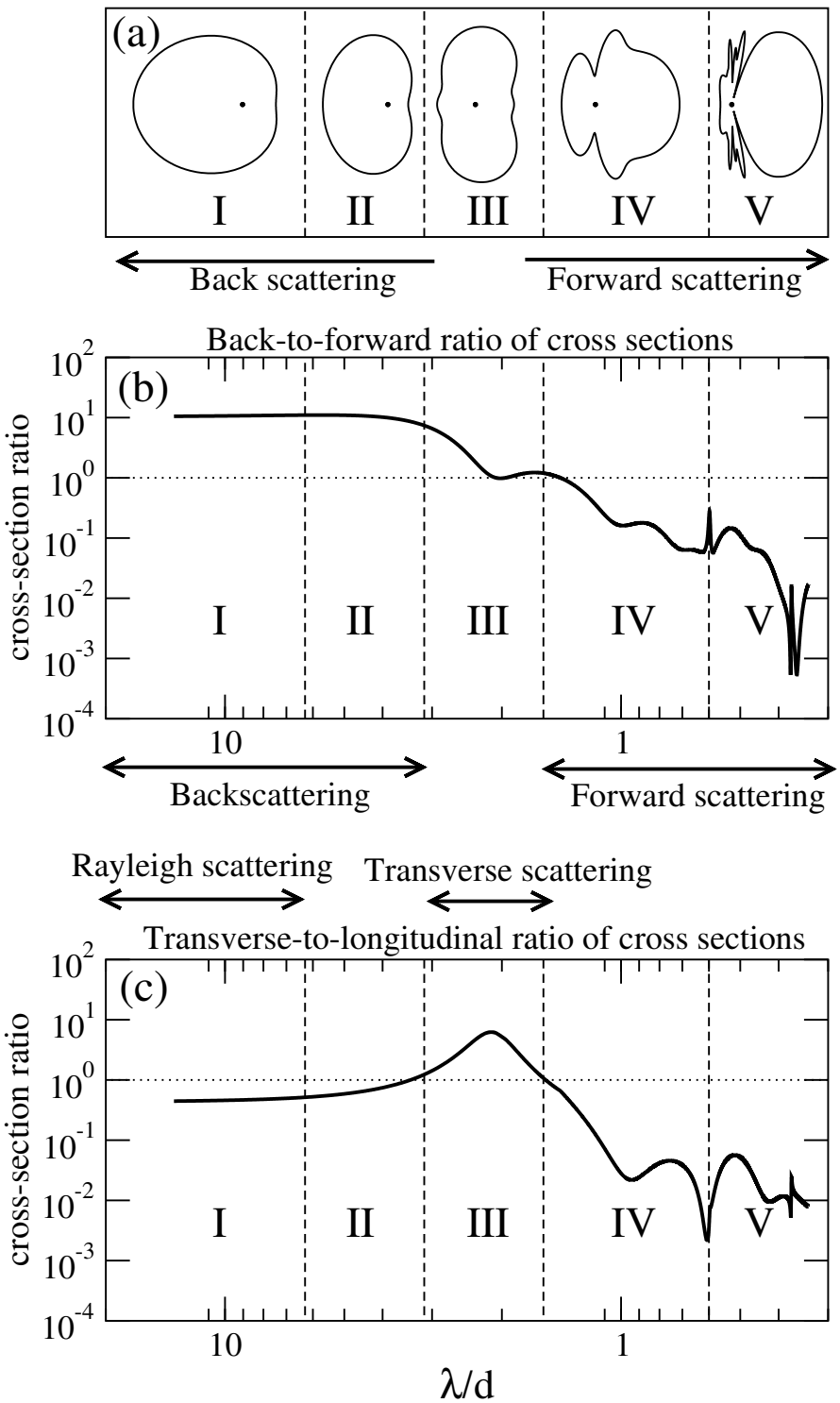

Fig. 6. a: Directivity pattern of a plane wave, coming from the right and incident on a spherical inclusion: the ration between the incident wavelength $\lambda$ and the scatterer diameter $D$ is given in terms of the study parameter $r=\lambda / D$. The directions of the backscattering and forward scattering are the rightward and leftward, respectively. The dashed lines represent the experimental frequency limits defining the frequency ranges noted $I, I I, I I I, I V$ and $V$. b: Back-to-forward ratio of scattering amplitude defined as $|f(0)|^{2} /|f(\pi)|^{2}$. The bands $I$ and $I I$ and the bands $I V$ and $V$ correspond to dominant back scattering and to dominant forward scattering, respectively. c: Transverse-to-longitudinal ratio of scattering amplitude defined as $|f(\pi / 2)|^{2} / \max \left[|f(\pi)|^{2},|f(0)|^{2}\right]$. The band $I I I$ corresponds to the frequency domain where transverse scattering is dominant. 


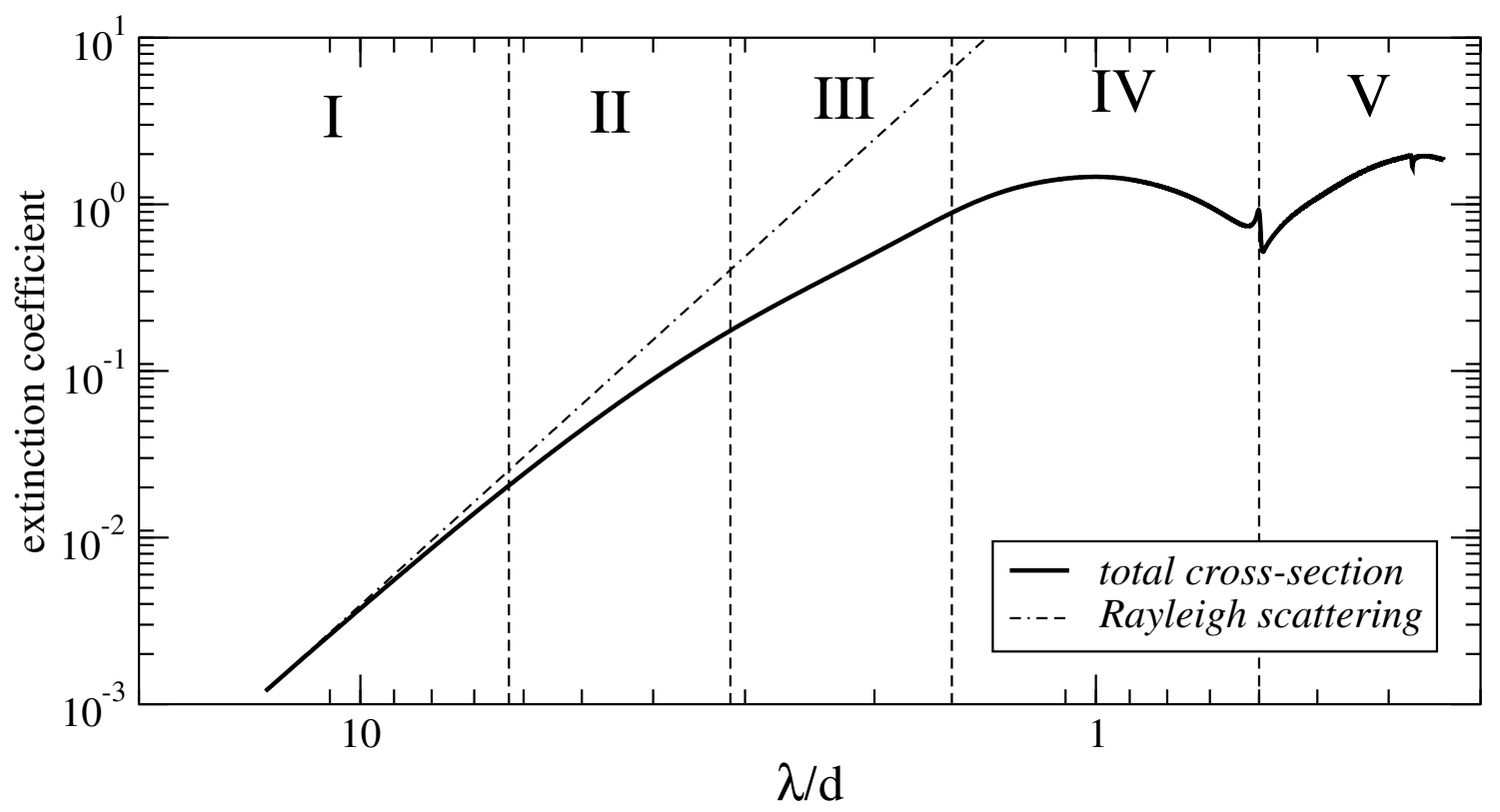

Fig. 7. Extinction coefficient (solid line), i.e. total scattering cross-section divided by the geometrical cross section $\pi d^{2} / 4$. Also shown are the five frequency bands identified in the data (see Figure 5). In band $I$, the scattering cross-section closely follows the Rayleigh scattering power law $\left(\propto \lambda^{-4}\right)$ (dashed line). 